\title{
Disseminated Carcinomatosis of the Bone Marrow from Occult Breast Cancer Responding to a Sequence of Endocrine Therapy
}

\author{
Takeshi Yamaguchi $^{\mathrm{a}}$ Mariko Masumoto ${ }^{\mathrm{a}} \quad$ Urara Sakurai $^{\mathrm{b}}$ \\ Minoru Nakane ${ }^{a}$ \\ aDivision of Medical Oncology, Japanese Red Cross Musashino Hospital, Tokyo, Japan; \\ bivision of Pathology, Japanese Red Cross Musashino Hospital, Tokyo, Japan
}

\begin{abstract}
Keywords
Unknown primary cancer - Occult breast cancer · Bone marrow metastasis - Endocrine therapy $\cdot$ CDK4/6 inhibitor
\end{abstract}

\begin{abstract}
Patients with cancer of unknown primary (CUP) are generally treated with chemotherapy. Bone marrow involvement suggests an advanced stage, and CUP with disseminated carcinomatosis of the bone marrow (DCBM) appears to have a dismal prognosis. However, our case of CUP with DCBM was successfully treated with a sequence of endocrine therapy over a long period. A woman presenting with low back pain was found to have multiple bone metastasis without an identifiable primary tumor on imaging studies. Blood tests revealed anemia and thrombocytopenia. A bone marrow biopsy was performed and showed relatively uniform small cells, strongly positive for estrogen receptor and progesterone receptor expression. We considered chemotherapy to be risky due to bicytopenia and an aromatase inhibitor, letrozole, was initiated. The patient's symptoms and laboratory findings gradually improved and bone lesions almost disappeared on FDG-PET/CT after 1 year of treatment. After 2 years on letrozole, hemoglobin levels and platelet counts had been gradually decreasing. Although she had no symptoms and no significant changes were observed on a CT scan, disease progression was highly likely. Thus, second-line treatment with fulvestrant and palbociclib was commenced, and hemoglobin levels and platelet counts were restored to within the normal ranges. She currently continues to receive fulvestrant and palbociclib over a year later. CUP complicated with DCBM might be metastatic occult breast cancer, and endocrine therapy can be a valuable treatment option if tumors express hormone receptors.
\end{abstract}

(C) 2020 The Author(s).

Published by S. Karger AG, Basel 
Yamaguchi et al.: Endocrine Therapy for Occult Breast Cancer with Bone Marrow Involvement

\section{Introduction}

In spite of the increasingly sophisticated diagnostic workup, no primary site can be identified in some patients with metastatic tumors. Cancer of unknown primary (CUP) accounts for approximately $3-5 \%$ of all malignancies. Treatment for CUP is challenging, owing to its aggressiveness and the often widespread disease at initial presentation [1]. Platinum-based chemotherapy is commonly utilized but shows modest efficacy, and patients with CUP generally have a dismal prognosis with median overall survival of less than 1 year. CUP comprises a heterogeneous group of patients, of whom $10-15 \%$ are classified as favorable subsets based on clinicopathological features. They should be treated similarly to patients with potentially equivalent primary tumors with metastatic dissemination, with the possibility of achieving long-term disease control.

Occult breast cancer (OBC) is one of the favorable subsets. Typical OBC cases are women who have adenocarcinoma or poorly differentiated carcinoma in the axillary lymph nodes and who have no evident primary breast lesion. Although less common, other metastatic sites have been reported in several case reports [2-6]. Disseminated carcinomatosis of the bone marrow (DCBM) is diffuse infiltration of the bone marrow by malignant cells. DCBM is accompanied by hematologic disorders such as leukopenia, anemia, and thrombocytopenia. Previous studies have reported that bone marrow metastasis from solid tumors is frequently seen in stomach, prostate, and breast cancer [7, 8]. Only a few reports of OBC with DCBM are available in the literature $[3,5,6]$, and findings concerning the treatment and clinical course are scarce.

Here, we present a case of OBC with DCBM which responded well to a sequence of endocrine therapy (ET).

\section{Case Presentation}

A 59-year-old woman presented at the department of orthopedic surgery at a local hospital with general fatigue and low back pain. Her past medical history included hypertension and hyperlipidemia. She had no prior history of malignancies. Blood tests revealed anemia. A vertebral fracture at L1 possibly due to malignant disease was detected by magnetic resonance imaging (MRI). Multiple myeloma was suspected, and she was referred to the division of hematology at our hospital. At the first visit, blood tests showed anemia (hemoglobin level, $8.9 \mathrm{~g} / \mathrm{dL}$ ) and slight thrombocytopenia (platelet count, 131,000/ $\mu \mathrm{L}$ ). White blood cell count was normal. The levels of immunoglobulins were unremarkable, and no monoclonal protein was detected by serum or urine electrophoresis. Bone marrow aspiration was attempted but resulted in a dry tap; therefore, a bone marrow biopsy was performed. The result of the bone marrow biopsy showed poorly differentiated carcinoma. Multiple myeloma was ruled out; then a CT scan was ordered which showed multiple mixed osteoblastic and osteolytic bone metastases in the absence of an identifiable primary tumor. She was diagnosed with CUP and referred to our division.

A physical examination including breast and pelvis revealed no suspicious primary site. At that time 5 weeks after the first visit, a further decline of the hemoglobin and platelet count was seen (hemoglobin level, $6.9 \mathrm{~g} / \mathrm{dL}$; platelet count, 37,000/ $\mu \mathrm{L}$ ). Other laboratory studies revealed elevated alkaline phosphatase. Serum levels of CEA (51.7 ng/mL), CA15-3 (88.7 U/mL), NSE $(31.5 \mathrm{ng} / \mathrm{mL})$, and ProGRP $(31.5 \mathrm{pg} / \mathrm{mL})$ were elevated. Other tumor markers such as CA125, CA19-9, and SCC were within the normal ranges. Upper and lower endoscopies of the gastrointestinal tract as a workup for a primary site were negative. Mammography, breast ultrasonography, and breast MRI did not find any suspicious lesions. FDG-PET/CT showed uptake in multiple bone lesions including the spine, pelvis, femur, and humerus, but nowhere else (Fig. 1A). 
Yamaguchi et al.: Endocrine Therapy for Occult Breast Cancer with Bone Marrow Involvement
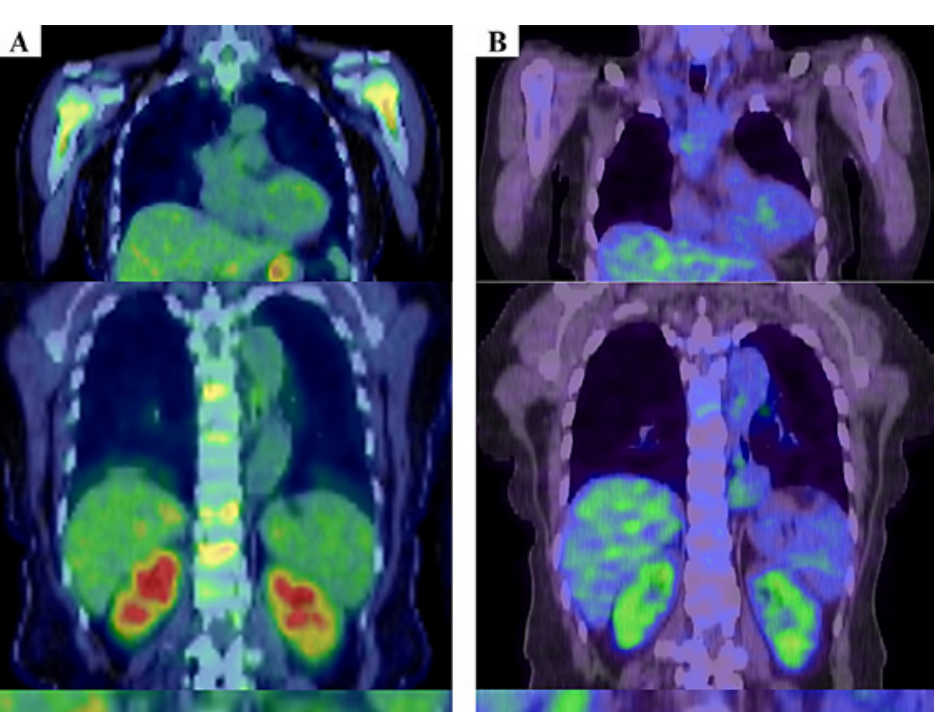

Fig. 1. FDG-PET/CT. FDG uptake was detected in multiple bones (humerus, spine, pelvis, femur) before treatment (A). Markedly decreased bone uptake was observed after 1 year of letrozole (B).
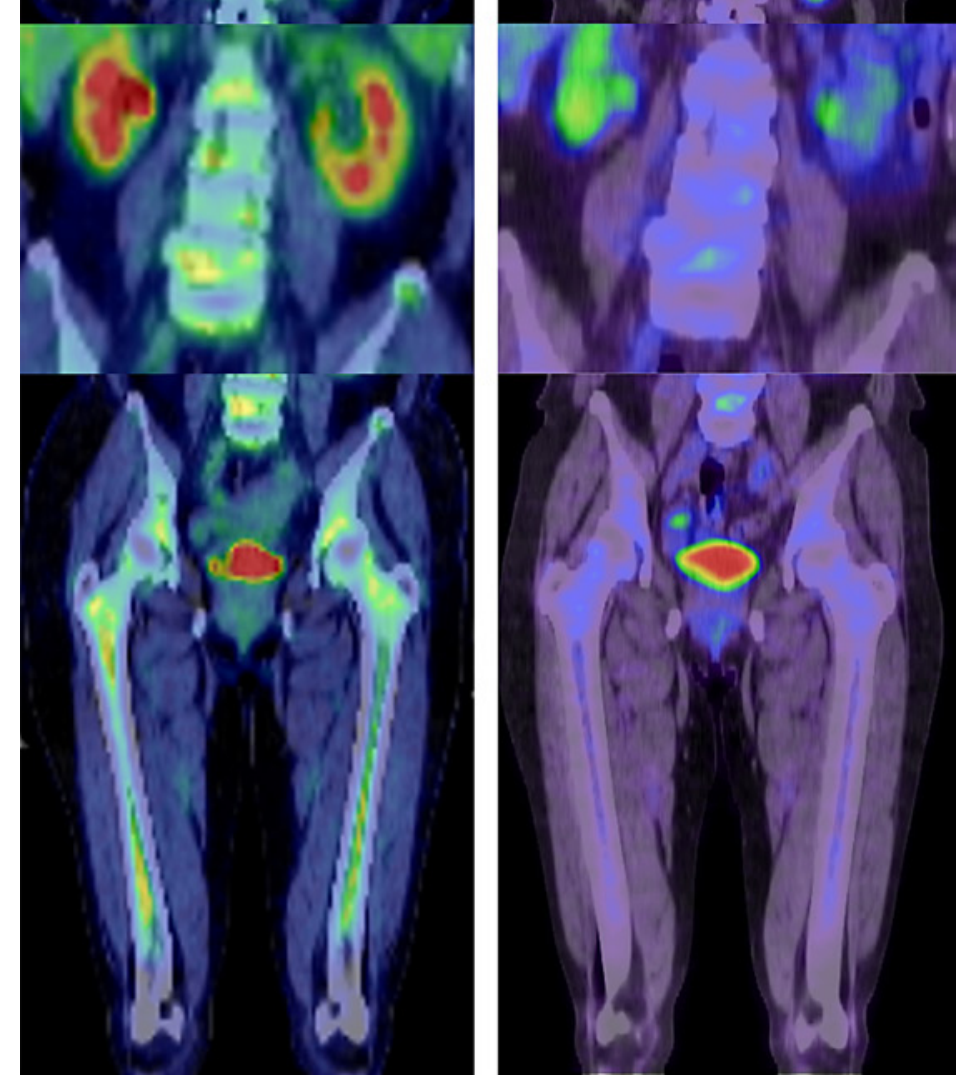

The bone marrow biopsy specimen was composed of relatively uniform small cells with scant cytoplasm and round hyperchromatic nuclei arranged in trabeculae and nests (Fig. 2A). The immunohistochemical (IHC) test proved positive for CK7 and synaptophysin (Fig. 2B, C), and negative for CK20, TTF-1, napsin A, CDX-2, chromogranin A, and CD56. Bone marrow involvement is associated with bone metastases in breast cancer, particularly with estrogen receptor (ER)-positive tumors [8]. Although breast examinations and FDG-PET/CT did not detect any lesions in the breast or axilla, additional IHC assays for markers of breast cancer were performed. The results were strongly positive for ER and progesterone receptor (PgR) (Fig. 2D, E), focally positive for E-cadherin, and negative for HER2 and GCDFP-15. The Ki-67 

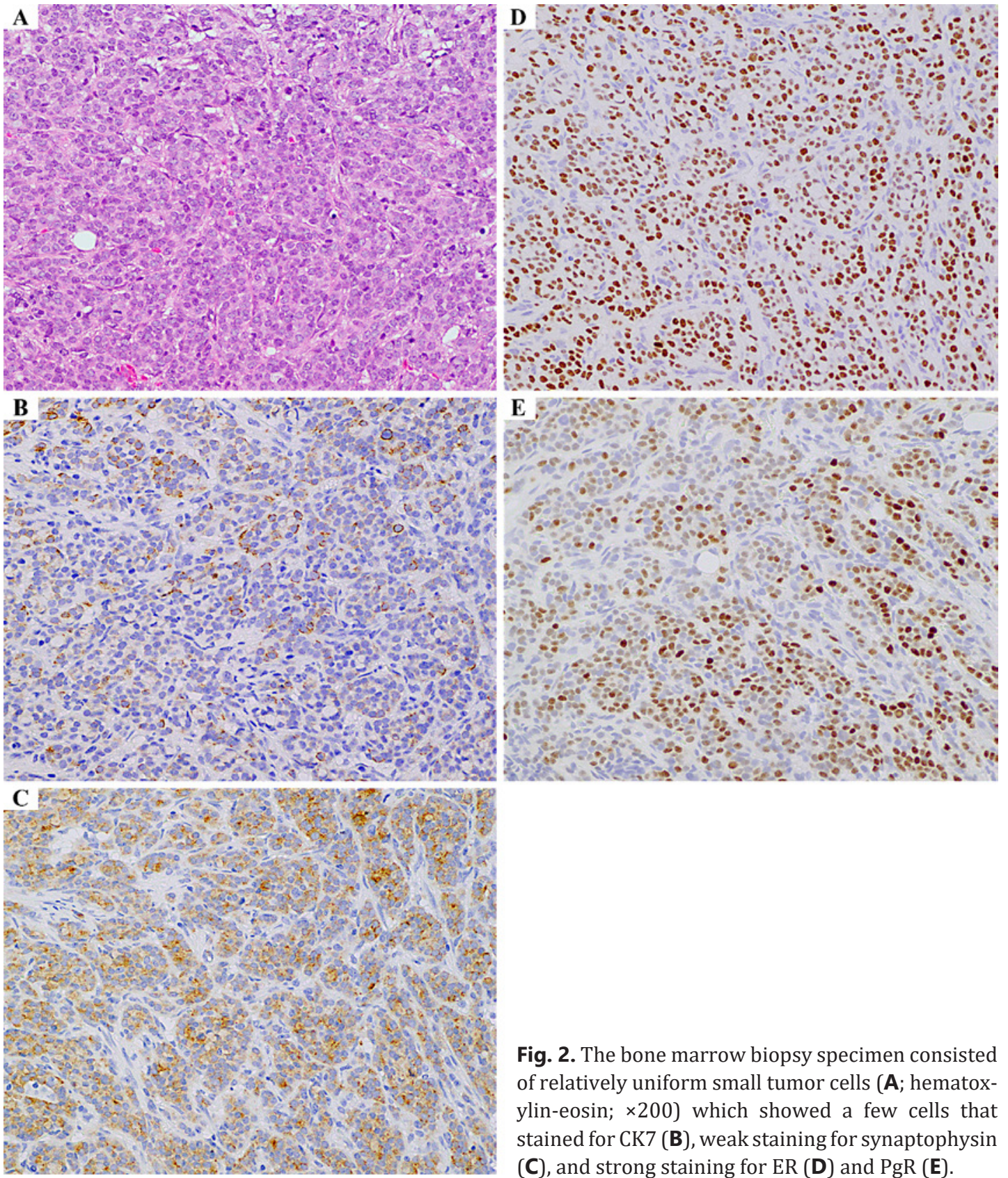

Fig. 2. The bone marrow biopsy specimen consisted of relatively uniform small tumor cells $(\mathbf{A}$; hematoxylin-eosin; $\times 200$ ) which showed a few cells that stained for CK7 (B), weak staining for synaptophysin (C), and strong staining for ER (D) and PgR (E).

labeling index was low (5-10\%). Staining for mammaglobin and GATA3 was not performed. Administration of chemotherapy carried a substantial risk due to the low platelet count. Instead, we decided to initiate treatment with an aromatase inhibitor, letrozole, because the tumors were hormone receptor (HR) positive. Radiotherapy was delivered to the lumbar vertebral metastases that caused pain and canal stenosis (30 Gy in 10 fractions), and denosumab was also started.

The patient's anemia and thrombocytopenia gradually improved after a month of letrozole (hemoglobin level, $8.2 \mathrm{~g} / \mathrm{dL}$; platelet count, 93,000/ $\mu \mathrm{L}$ ). After 4 months of treatment, her blood count was restored to within the normal range. FDG-PET/CT performed after a year of letrozole treatment demonstrated that most of the uptake into bone metastases, which was 
Yamaguchi et al.: Endocrine Therapy for Occult Breast Cancer with Bone Marrow Involvement

observed before treatment, was no longer visible (Fig. 1B), which suggested that the letrozole treatment was efficacious.

After 2 years on letrozole, the patient's hemoglobin levels and platelet counts had continued to gradually decrease (hemoglobin level, $7.1 \mathrm{~g} / \mathrm{dL}$; platelet count, 91,000/ $\mu \mathrm{L}$ ). Alkaline phosphatase levels were within the normal range. She had no symptoms. A CT scan did not show any significant changes from previous assessment. FDG-PET/CT could not be performed promptly. Disease progression was highly likely from the recurrence of hematologic abnormalities and so second-line treatment with fulvestrant and a reduced dose of palbociclib (75 mg/day) was commenced. Platelet counts dropped down to $41,000 / \mu \mathrm{L}$ after 2 weeks of palbociclib, so palbociclib was interrupted and fulvestrant alone was continued. Two months after treatment initiation, platelet counts rose to $100,000 / \mu \mathrm{L}$ and palbociclib was restarted. The normalization of platelet counts and hemoglobin levels was achieved 3 and 6 months, respectively, after fulvestrant and palbociclib treatment commenced. The patient currently continues the second-line treatment and has no symptoms. No new lesions have been detected by CT scan so far.

\section{Discussion}

Our patient's tumor cells expressed HR, and treatment with ET was found to be highly effective; thus, we believed the current case was metastatic OBC with a rare clinical presentation. The first-line ET was effective. At progression, the patient was successfully salvaged with the second-line ET combined with a CDK4/6 inhibitor. Although a diagnosis of DCBM is generally regarded as an indicator of poor prognosis, our patient has survived over 3 years so far. There are no previous reports describing successful treatment of metastatic OBC presenting with DCBM with ET including a CDK4/6 inhibitor and where long-term disease control was achieved.

Although a CK7/20 immunophenotype was consistent with breast cancer, GCDFP-15, a diagnostic IHC marker of breast cancer, was negative. However, its sensitivity ranges between 5 and 74\% [9]. It is regrettable that we could not evaluate GATA-3 expression which has been reported to show higher sensitivity than GCDFP-15 [10]. Positive IHC staining for synaptophysin suggested a neuroendocrine tumor. They usually arise from the lungs or gastrointestinal tract but can also derive from the breast on rare occasions. Neuroendocrine carcinoma of the breast accounts for $2-5 \%$ of breast carcinomas. Neuroendocrine carcinoma of the breast is often positive for HR, and HER2 is almost always negative, as was seen in our case [11].

Treatment of patients with DCBM is challenging because of cytopenia. Systemic chemotherapy is generally used and recommended for DCBM in breast cancer $[8,12,13]$. However, chemotherapy can lead to the exacerbation of pretreatment hematologic disorders from DCBM and thus the need for supportive therapy including blood transfusion and granulocyte colony-stimulating factor support. On the other hand, endocrine agents do not have myelotoxicity. Positive HR status does not necessarily guarantee responsiveness to ET in CUP, since these markers are not sufficiently specific to breast cancer [14]. Nevertheless, treatment with ET should be considered in HR-positive CUP because it has low toxicity and is generally not harmful.

Studies concerning the efficacy of ET in OBC with DCBM are very limited. Only one case report by Fan et al. [6] described the use of tamoxifen alone which achieved a sustained response. They did not report the clinical course after progression on tamoxifen. Our case was treated with letrozole because aromatase inhibitors have been shown to be superior to tamoxifen in postmenopausal women with breast cancer. The response to letrozole lasted for 
Yamaguchi et al.: Endocrine Therapy for Occult Breast Cancer with Bone Marrow Involvement

2 years and no skeletal-related events occurred thanks to the concurrent use of denosumab. The second-line treatment with fulvestrant and palbociclib also induced an impressive response. There are three commercially available CDK4/6 inhibitors (palbociclib, abemaciclib, and ribociclib) for metastatic breast cancer. The randomized phase III trials of CDK4/6 inhibitors have shown prolongation of progression-free survival with the addition of a CDK4/6 inhibitor to an endocrine agent alone in the first-line and second-line setting. An overall survival benefit has recently also been demonstrated in some trials [15]. The efficacy and safety of CDK4/6 inhibitors in patients with DCBM have not been elucidated because patients with bone marrow involvement are generally excluded from clinical trials. We chose to start with the lowest dose level of palbociclib (75 mg/day) because we were concerned about myelosuppression. Nevertheless, NCI-CTCAE grade 3 thrombocytopenia occurred and we temporarily interrupted palbociclib. Abemaciclib has the lowest incidence of neutropenia among CDK4/6 inhibitors. Because DCBM causes cytopenias, abemaciclib seems to be the preferred drug. Unfortunately, we were not able to access it at the time of progression.

Previous studies showed that DCBM in breast cancer is usually observed in HR-positive tumors $[8,12,13]$. When patients present with DCBM but without an identifiable primary site, staining IHC panels for breast cancer should be considered. If chemotherapy is deemed risky due to cytopenias, ET and CDK4/6 inhibitors can be a valuable treatment option when tumors express HR.

\section{Statement of Ethics}

The patient gave written informed consent.

\section{Disclosure Statement}

The authors have no conflicts of interest to declare.

\section{Funding Sources}

The authors have no funding source to report.

\section{Author Contributions}

T. Yamaguchi wrote the main manuscript and other authors critically reviewed it. All authors were involved in the patient's clinical management.

\section{References}

1 Varadhachary GR, Raber MN. Cancer of unknown primary site. N Engl J Med. 2014;371(8):757-65.

2 Neal L, Sookhan N, Reynolds C. Occult breast carcinoma presenting as gastrointestinal metastases. Case Rep Med. 2009;2009:564756.

3 Mahdi EJ, Mahdi AJ. Leucoerythroblastosis and thrombocytopenia as clues to metastatic malignancy. BMJ Case Rep. 2014;2014. pii: bcr2013202612.

4 Weimann ET, Botero EB, Mendes C, Santos MA, Stelini RF, Zelenika CR. Cutaneous metastasis as the first manifestation of occult malignant breast neoplasia. An Bras Dermatol. 2016;91(5 Suppl 1):105-7. 
5 Liu L, Zhang J, Chen M, Ren S, Liu H, Zhang H. Anemia and thrombocytopenia as initial symptoms of occult breast cancer with bone marrow metastasis: a case report. Medicine (Baltimore). 2017;96(45):e8529.

6 Fan FS, Yang CF, Wang YF. Diffuse bone marrow metastasis as the initial presentation of an occult breast cancer. Case Rep Oncol Med. 2018;2018:2946409.

7 Delsol G, Guiu-Godfrin B, Guiu M, Pris J, Corberand J, Fabre J. Leukoerythroblastosis and cancer frequency, prognosis, and physiopathologic significance. Cancer. 1979;44(3):1009-13.

8 Kopp HG, Krauss K, Fehm T, Staebler A, Zahm J, Vogel W, et al. Symptomatic bone marrow involvement in breast cancer-clinical presentation, treatment, and prognosis: a single institution review of 22 cases. Anticancer Res. 2011;31(11):4025-30.

9 Gown AM, Fulton RS, Kandalaft PL. Markers of metastatic carcinoma of breast origin. Histopathology. 2016; 68(1):86-95.

10 Ni YB, Tsang JYS, Shao MM, Chan SK, Cheung SY, Tong J, et al. GATA-3 is superior to GCDFP-15 and mammaglobin to identify primary and metastatic breast cancer. Breast Cancer Res Treat. 2018;169(1):25-32.

11 Inno A, Bogina G, Turazza M, Bortesi L, Duranti S, Massocco A, et al. Neuroendocrine carcinoma of the breast: current evidence and future perspectives. Oncologist. 2016;21(1):28-32.

12 Demir L, Akyol M, Bener S, Payzin KB, Erten C, Somali I, et al. Prognostic evaluation of breast cancer patients with evident bone marrow metastasis. Breast J. 2014;20(3):279-87.

13 Shinden Y, Sugimachi K, Tanaka F, Fujiyoshi K, Kijima Y, Natsugoe S, et al. Clinicopathological characteristics of disseminated carcinomatosis of the bone marrow in breast cancer patients. Mol Clin Oncol. 2018;8(1):93-8.

14 Kandalaft PL, Gown AM. Practical applications in immunohistochemistry: carcinomas of unknown primary site. Arch Pathol Lab Med. 2016;140(6):508-23.

15 Preusser M, De Mattos-Arruda L, Thill M, Criscitiello C, Bartsch R, Ruhstaller T, et al. Correction: CDK4/6 inhibitors in the treatment of patients with breast cancer: summary of a multidisciplinary round-table discussion. ESMO Open. 2019;4(2):e000368corr1. 